\title{
Comparison of PSORI-CM01 granules and Yinxieling tablets for patients with chronic plaque psoriasis: a pilot study for a randomized, double-blinded, double-dummy, multicentre trial
}

\author{
Dan-Ni Yao, ${ }^{1,2}$, Chuan-Jian Lu ${ }^{1,2,3,4,5}$, Ze-Huai Wen ${ }^{6,7}$, Yu-Hong Yan ${ }^{1,2}$, Li-Ming Lu ${ }^{8}$, Hui-Mei Wu ${ }^{1}$, \\ Zi-Yang $\mathrm{He}^{1}$, Hao Deng ${ }^{1,2}$, Jing-Wen Deng ${ }^{1,2}$
}

${ }^{1}$ Department of Dermatology, Guangdong Provincial Hospital of Chinese Medicine, Guangzhou, China; ${ }^{2}$ Guangdong Provincial Academy of Chinese Medical Sciences, Guangzhou, China; ${ }^{3}$ Guangdong Provincial Key Laboratory of Clinical Research on Traditional Chinese Medicine Syndrome, Guangzhou, China; ${ }^{4}$ State Key Laboratory of Dampness Syndrome of Chinese Medicine; ${ }^{5}$ Guangdong-Hong Kong-Macau Joint Lab on Chinese Medicine and Immune Disease Research, Guangzhou China; ${ }^{6}$ Key Unit of Methodology in Clinical Research, Guangdong Provincial Hospital of Chinese Medicine, Guangzhou, China; ${ }^{7}$ National Centre for Design Measurement and Evaluation of Clinical Research, Guangzhou University of Chinese Medicine, Guangzhou, China; ${ }^{8}$ Clinical Research and Data Center, South China Research Center for Acupuncture and Moxibustion, Medical College of Acu-Moxi and Rehabilitation, Guangzhou University of Chinese Medicine, Guangzhou, China

Contributions: (I) Conception and design: CJ Lu, DN Yao, ZH Wen; (II) Administrative support: CJ Lu, DN Yao; (III) Provision of study materials or patients: DN Yao, HM Wu, ZY He, H Deng, YH Yan; (IV) Collection and assembly of data: HM Wu, JW Deng; (V) Data analysis and interpretation: DN Yao, ZH Wen, LM Lu; (VI) Manuscript writing: All authors; (VII) Final approval of manuscript: All authors.

Correspondence to: Chuan-Jian Lu. Guangdong Provincial Hospital of Chinese Medicine, No. 111 Dade Road, Yuexiu District, Guangzhou 510120, China. Email: 1cj@gzucm.edu.cn.

Background: To compare the efficacy and safety of PSORI-CM01 granules with Yinxieling tablets in patients with chronic plaque psoriasis (CPP), we plan to conduct a multicentre, randomized, double-blinded, double-dummy, controlled trial. This pilot study was conducted to determine the feasibility and the potential of the protocol for the full-scale randomized controlled trial (RCT).

Methods: This pilot study was conducted in three centers, and compared PSORI-CM01 granules with Yinxieling tablets in patients with CPP during a 12-week treatment and 3-month follow-up period. The primary efficacy endpoint was the decrease of the psoriasis area severity index (PASI) at week 12 . The secondary outcome measures included reduction rates of PASI, pruritus scores on the Visual Analogue Scale (VAS), body surface area (BSA), and the Dermatology Life Quality Index (DLQI). Safety was assessed via the incidence of adverse events (AEs) in each treatment group.

Results: A total of 211 patients were screened, and 63 subjects who met the inclusion criteria were randomised to PSORI-CM01 granule group (N=31) or Yinxieling tablets group (N=32) while 39 subjects finished the study. The primary outcome measure showed a mean decrease of PASI of 2.03 in the PSORICM01 group compared to 0.89 in the Yinxieling group at week 12. Except for the VAS score $(\mathrm{t}=-2.261$, $\mathrm{P}=0.027)$, the secondary outcomes showed no significant improvement from baseline in both groups at week 12. No safety or tolerability concerns related to the drugs were observed in either group.

Conclusions: This pilot study showed that the RCT is feasible for randomization, patient recruitment, and assessment. Major strategies are necessary to reduce the patient dropout rate before conducting the full RCT. In this pilot study, the PSORI-CM01 granule exhibited greater potential for development compared to its original formula (Yinxieling tablets) for the treatment of CPP.

Keywords: Psoriasis vulgaris; randomized double-blinded, double-dummy, controlled trial; PSORI-CM01 granule; Chinese herbal medicine; Yinxieling tablet

$\wedge$ ORCID: 0000-0002-6507-3505. 
Submitted Oct 26, 2020. Accepted for publication Jan 21, 2021.

doi: 10.21037/apm-20-2575

View this article at: http://dx.doi.org/10.21037/apm-20-2575

\section{Introduction}

Psoriasis is a chronic disease that ebbs and flows during a patient's lifetime without curative treatment. Plaque psoriasis is the most common form that affects approximately $80 \%$ to $90 \%$ of patients (1). For mild to severe psoriasis patients (2), it can often be managed with topical agents. However, due to its frequent relapse rates and long-term treatment, most topical agents (such as topical corticosteroids) are not considered appropriate for long-term use due to of their side effects.

Evidence has shown that Chinese herbal medicine (CHM) can relieve symptoms and reduce PASI scores with fewer side effects and toxicity $(3,4)$. Meta-analyses of clinical trials have suggested that at least some CHM treatments can offer clinical benefits (5-7). Yinxieling tablets, a Chinese herbal formula developed by Prof. Guowei Xuan (a wellknown dermatologist of Chinese medicine in China), have been used for over 20 years in the Guangdong Provincial Hospital of Chinese Medicine (GPHCM). Yinxieling is composed of angelica sinensis (danggui), radix paeoniae rubra (chi shao), chloranthus spicatus, smoked plum, radix rebmanniae recen, ligusticum wallichii, radices lithospermi, curcuma zedoary, rbizome smilacis glabrae, and liquorice (8), and is registered on Guangdong Food and Drug Administration (hospital preparation approval No. Z20080123). Previous clinical trials have demonstrated its ability to decrease the Psoriasis Area Severity Index (PASI) and serum levels of tumor necrosis factor alpha (TNF- $\alpha$ ) and interleukin-8 (IL-8) after 8 weeks of treatment, with fewer side effects $(8,9)$. According to previous studies, the Yinxieling formula also showed inhibitory effects on proliferating cell nuclear antigen (PCNA) of vaginal epithelium in mice, which in turn promoted an apoptotic effect on keratinocytes (10). However, considering that it is composed of 10 kinds of Chinese herbs, its development into a new drug is difficult.

Based on the traditional Chinese theory and the rule for further optimization of the prescription, we optimized Yinxieling into PSORI-CM01 (an optimized Yinxieling formula) with only seven ingredients (radix paeoniae rubra, smoked plum, chloranthus spicatus, radices lithospermi, curcuma zedoary, rhizome smilacis glabrae, and liquorice), and with an orthogonal design $(11,12)$. This optimization technique has been recognized and obtained a national patent (patent number: ZL 200910091066.5). Randomized controlled trials (RCTs) of PSORI-CM01 have shown a decrease in the PASI and Dermatology Life Quality Index (DLQI) scores, and no adverse events (AEs) were reported during the study period (4). Another 12-week observational study showed that PASI of patients with psoriasis was reduced after treatment with PSORI-CM01, and the metabolic variations were visualized in patients with psoriasis before and after PSORI-CM01 treatment (13). Compared with the normal saline group, the PSORI-CM01 decoction could significantly inhibit the mitosis of mouse vaginal epithelium and promote the formation of mouse tail scale epidermis. It could also inhibit $\mathrm{HaCaT}$ cell growth after 24 hours of treatment (14).

However, the efficacy and safety of the optimized formula (PSORI-CM01) compared to its original formula (Yinxieling) is still unknown. From the similar results of the previous study, we have shown that the efficacy of the new formula is superior to the old one. If this hypothesis is true, the new formula, which contains fewer ingredients, could lead to fewer adverse effects, reduce the cost, and have greater availability for application compared to the original formula. To investigate this, we designed a RCT that was registered on the $8^{\text {th }}$ August 2014 (ChiCTRTRC-14005185). The aim of this pilot study was to determine the feasibility of the protocol and assess the potential for a full-scale RCT (15). We report the results of this pilot trial in the article and present the following article in accordance with the CONSORT reporting checklist (available at http://dx.doi.org/10.21037/apm-20-2575).

\section{Methods}

\section{Design and eligibility}

A multicentre, prospective, randomized, double-blind, double-dummy, and parallel-group pilot study was designed to compare the optimized formula of PSORI-CM01 granules with its original formula, Yinxieling tablets. A randomized number was generated by the computer, using the blocked randomization method with a block size of 4 and a ratio of $1: 1$, created by SAS 9.2 software (SAS 
Institute Inc., Cary, USA) and performed by the Key Unit of Methodology in Clinical Research (KUMCR) of the GPHCM. This was achieved through an Interactive Web Response System for Chinese Medicine Trials (IWRSCMT), which was a verified online randomization facility established by the KUMCR (http://www.gztcmgcp.com/ sjxt/login.asp). The results of randomized allocation from the GPHCM were preserved by the KUMCR until the analysis was completed. The Yinxieling tablets and PSORICM01 granules are present in different dosage forms; to guarantee a double-blinded method we used placebo granules and tablets in the two groups. The participants, paramedics, investigators, outcome assessors, statisticians, and other staff who helped to dispense medications were all blinded to the allocation. All procedures performed in this study involving human participants were in accordance with the Declaration of Helsinki (as revised in 2013). The study protocol was approved by the Institutional Ethics Committee of GPHCM (Ethics Statement No.: B2014026-01, approved on $8^{\text {th }}$ August 2014), the Biomedical Ethics Committee of the Hangzhou No. 3 General Hospital (Ethics Statement No.: B2014-026-01, approved on $8^{\text {th }}$ August 2014), and the Institutional Ethics Committee of the Affiliated Hospital of Tianjin Academy of Traditional Chinese Medicine (Ethics Statement No.: 2014-KY-001). All patients provided written informed consent prior to initiation of the study.

\section{Patients and setting}

Patients were mainly recruited by dermatologists or investigators from dermatology outpatient clinics in the GPHCM, the Affiliated Hospital of Tianjin Academy of Traditional Chinese Medicine, and the Hangzhou No. 3 General Hospital, or through local newspapers or posters. Participants were diagnosed with psoriasis vulgaris (PV) by dermatologists and were selected according to the inclusion and exclusion criteria. The inclusion criteria were as follows: (I) patients diagnosed with PV $[3<$ PASI $<30$ and body surface area (BSA) $<30 \%$ ]; (II) patients that provided written/signed informed consent; (III) patients aged between 18 and 65 years.

Patients were excluded based on the following criteria: (I) guttate psoriasis, inverse psoriasis, or psoriasis that was exclusively associated with the face; (II) acute progression of psoriasis, and erythroderma; (III) current (or within 1 year) pregnancy or lactation; (IV) current clinically significant anxiety or depression with a Self-rating Anxiety Scale (SAS) score of $>50$ or a Self-rating Depression Scale (SDS) score of $>53$, or with other psychiatric disorders; (V) history of primary cardiovascular, respiratory, digestive, urinary, endocrine, and hematologic diseases, which cannot be controlled through ordinary treatments; history of known malignancies, infection, electrolyte imbalance, and acidbase disturbance. Patients with clinical test results listed below: AST (Aspartate aminotransferase) or ALT (Alanine aminotransferase) three times higher than the upper limit of normal; creatinine 1.5 times higher than upper limit of normal; elevation of hemoglobin $20 \mathrm{~g} / \mathrm{L}$ higher than upper limit of normal; platelet count of less than $75.0 \times 10^{9} / \mathrm{L}$; white blood cell count of less than $3.0 \times 10^{9} / \mathrm{L}$; any other abnormal laboratory test results, as assessed by the investigators, that are not suitable for this clinical study; (VI) allergy to any medicine or ingredients used in this study; (VII) current registration in other clinical trials or participation within 1 month; (VIII) topical treatments (i.e., corticosteroids or retinoic acid) used within 2 weeks; systemic therapy or phototherapy (ultraviolet radiation $\mathrm{B}, \mathrm{UVB}$ ) and psoralen combined with ultraviolet A (PUVA) within 4 weeks or biological therapy within 12 weeks; (IX) systemic treatment prescribed by doctors.

For participant selection, we registered every patient with psoriasis who attended to see a dermatologist or who came to consult dermatologists or investigators regarding clinical trials. Investigators introduced the trial to them and obtained their written/signed informed consent. Patients who were willing to participate the trial had to be evaluated for some indicators, including PASI, SAS, and SDS scores. Patients who were eligible following their initial assessment were required to undergo a 2 -week run-in period.

All patients had a run-in period of 2 weeks before randomization. During the run-in period, all patients needed to cease all previous treatments except for urea cream. Subjects were screened again according to the inclusion and exclusion criteria at the end of the runin period. Laboratory tests and electrocardiograms were monitored at baseline after that. Eligible subjects were assigned according to their sequential numbers, which corresponded to a random number, and were then randomized into one of the two groups (see Figure 1).

\section{Intervention}

Patients were randomized (1:1) into either the PSORICM01 group or the Yinxieling group. Patients in the PSORI-CM01 group received PSORI-CM01 granules 


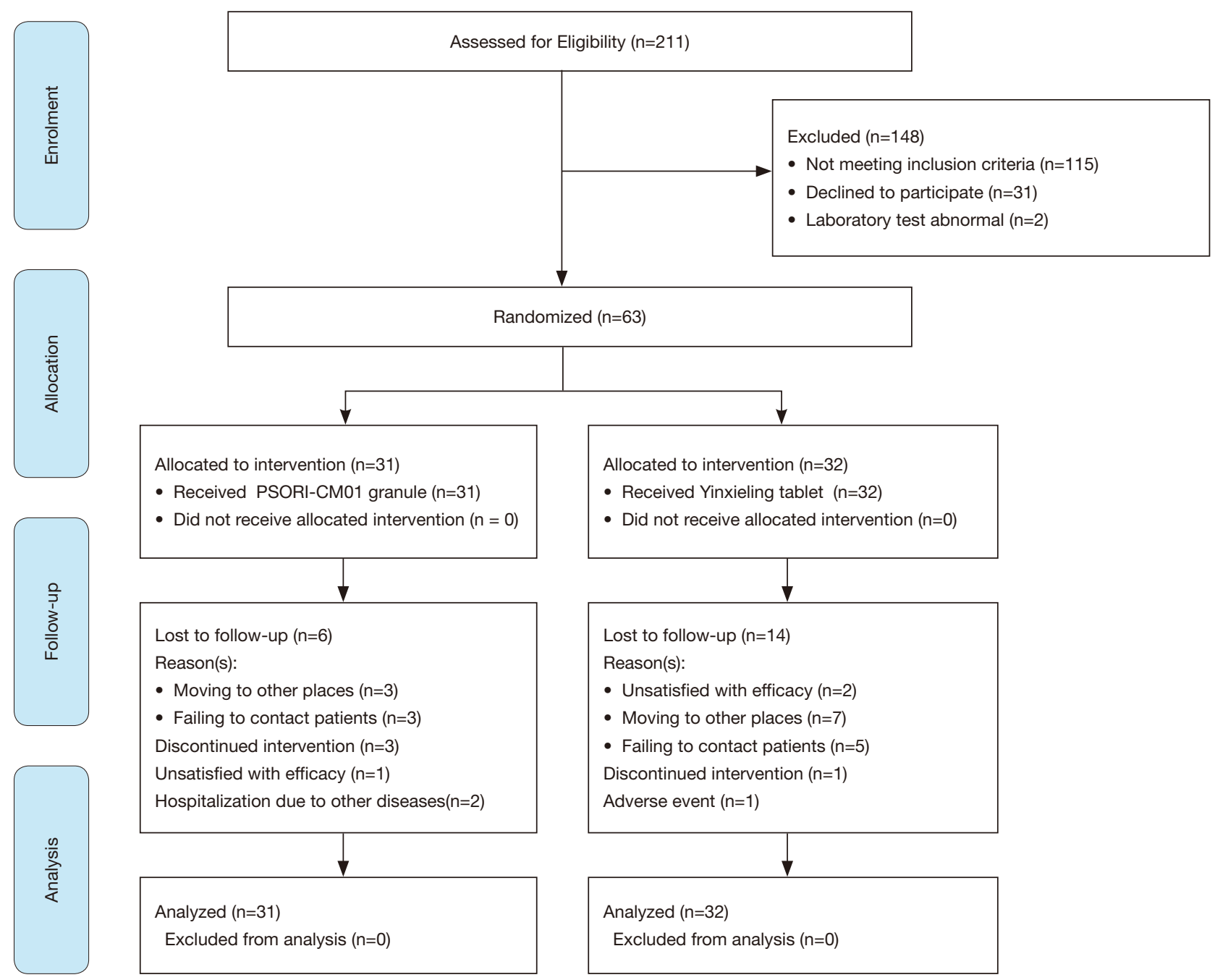

Figure 1 Flowchart of the trial.

(twice daily) and five placebo tablets (three times daily) after meals for 12 weeks. Patients in the Yinxieling group received placebo granules (twice daily) and five Yinxieling tablets (three times daily) after meals for 12 weeks. PSORICM01 granules and matching placebo granules used in the trial were manufactured by the Tianjiang Pharmaceutical Co. Ltd. (Jiangyin, Jiangsu Province, China). Yinxieling tablets and matching placebo tablets were manufactured by Kangyuan Pharmaceutical Co. Ltd. (Guangzhou, Guangdong Province, China). Both of these companies met the requirements of Good Manufacturing Practice (GMP). The matching placebo granule (main ingredients: maltodextrin, lactose, and natural edible pigment) was similar to the PSORI-CM01 granule in appearance, weight, and taste. Also, the matching placebo tablet (main ingredients: maltodextrin, lactose, and natural edible pigment) was similar to the Yinxieling tablet in appearance, weight, and taste. The quality control of the PSORI-CM01 granule was accredited by the Guangdong Provincial Laboratory using liquid chromatography coupled with an LTQ Orbitrap mass spectrometer (16), and the quality control of Yinxieling Tablet was accredited by the quality certificate of analysis. After 12 weeks of treatment, patients were followed up for 3 months in the follow-up period without intervention until they relapsed. Relapse was defined as a loss of $50 \%$ of PASI improvement from baseline in patients who achieved treatment success (which was defined as at least $50 \%$ improvement in the PASI score 
from baseline) $(17,18)$. According to the doctors' opinions, urea ointment was used as the basic concomitant treatment during the run-in and follow-up periods. If serious itching occurred, cetirizine hydrochloride $(10 \mathrm{mg} /$ day $)$ would be taken as the concomitant medication. The flowchart of the study is illustrated in Figure 1.

\section{Outcome measures}

The primary efficacy outcome measure was the difference in the PASI score after 12 weeks of treatment. PASI scores vary from 0.0 (no lesions at all) to 72.0 (complete erythroderma of the most severe degree possible).

Secondary outcome measures in the study were as follows: (I) reduction rate of PASI after treatment; (II) pruritus scores on the Visual Analogue Scale (VAS); (III) improvement in BSA for PV; (IV) improvement in DLQI; and $(\mathrm{V})$ incidence of serious adverse events (SAEs).

PASI and BSA were assessed every 2 weeks during the treatment period and every 4 weeks in the follow-up period. Meanwhile, patients were required to report VAS scores and to keep diaries to record any emergencies that occurred at any time during the study period. DLQI was calculated every 4 weeks.

Safety was measured based on the incidence of AEs and the patient's vital signs (heart rate and blood pressure). Laboratory tests including complete blood count, urinalysis, hepatic and renal functions, and electrocardiogram were monitored and recorded at baseline and at 12 weeks after treatment.

\section{Sample size}

For the large-scale study, we calculated sample size based on our previous study using PASW Statistical software (version 18.0; IBM Inc., Chicago, IL, USA). We assumed that the effectiveness of the PSORI-CM01 granules would be a PASI improvement growth of more than 1.5 times compared to the Yingxieling tablets. Group sample sizes of 236 per group. And 236 per group achieved an $80 \%$ power to detect superiority using a one-sided, two-sample $t$-test. The margin of equivalence was 1.0. The significance level (alpha) of the test was 0.025 . The data are drawn from populations with standard deviations of 1.1 and 2.5. Assuming a $15 \%$ drop-out rate, at least 556 patients in total, (278 in each group) had been recruited.

However, sample size calculations are not a prerequisite for pilot studies (19). In this trial, 63 patients were recruited according to the recommendations for pilot trials to test the practicality of the trial design

\section{Statistical analysis}

The following populations were considered for analysis: (I) the intention-to-treat (ITT) population, which included all randomized patients who received at least one dose of study treatment and the data of at least one visit; (II) the per-protocol (PP) population, which included all subjects from the ITT population who completed the treatment period, with a medication compliance of between $80 \%$ and $120 \%$, and without any major protocol deviations; and (III) the safety population, which included all randomized patients who received at least one dose of study treatment and at least one laboratory test after the treatment. Missing values were replaced by the last observation carried forward (LOCF) method for the ITT analyses.

Data were processed using PASW Statistics 18.0 (IBM SPSS Inc., Armonk, New York, USA). Descriptive analysis was used to analyze patients' clinical demographics. Data were presented as mean and standard deviation at each time point. The $t$-test was used to compare the primary outcome between two groups at the end of treatment. The $t$-test, Mann-Whitney U test, and Fisher's exact test were used to compare secondary outcomes, such as BSA and VAS, at the end of treatment. Two-tailed $\mathrm{P}$ values $<0.05$ were considered statistically significant.

The main objective of this pilot trial was to test the practicality of the trial design. So, it is inappropriate to make any inferences or state findings of efficacy, as this was a pilot study, as opposed to a hypothesis test (20). Therefore, only data from the pilot trial were analyzed to alert investigators to issues with regards to testing in the planned larger-scale trial.

\section{Patient and public involvement}

The interventions in the trial were specifically designed based on clinical application of the two formulas and to meet the needs and experiences of participants (18-65 years). The outcome measures used in the surveys were all ageappropriate. In this trial, the burden of the intervention was assessed by investigators. A summary of the results will be provided to participants by public lectures after publication. Patients and the public were not involved in the design, 
conduct, reporting, or dissemination of plans of our research.

\section{Results}

\section{Patients}

Of the 211 recruited patients, 63 were screened and randomized to either the PSORI-CM01 group $(\mathrm{N}=31)$ or the Yinxieling group ( $\mathrm{N}=32)$ from the outpatient departments of three centers over a 3-year period. All patients received at least one dose of intervention and were considered evaluable for both safety and ITT efficacy analyses. Of them, 25 patients in PSORI-CM01 group and 22 patients in Yinxieling group completed the treatment for 12 weeks. Thirty-nine patients who completed the treatment (22 in the PSORI-CM01 group vs. 17 in the Yinxieling group) were followed up for 12 weeks after therapy. Nine patients in the PSORI-CM01 group and 15 patients in the Yinxieling group did not complete the study protocol due to premature discontinuation and/or major protocol deviations, and were therefore excluded from the PP analysis.

Table 1 summarizes the baseline characteristics of the 63 patients. The severity of psoriasis at baseline between patients who completed the study and those who dropped out did not differ significantly $(\mathrm{t}=0.964, \mathrm{P}=0.339)$. The mean PASI score at baseline was 6.41 in the PSORICM01 group compared with 5.84 in the Yinxieling group. Baseline severity of psoriasis ranged from mild to severe with individual PASI scores of up to 14. The results showed that the groups were similar in size, gender, age, and other characteristics (such as medical history) at baseline (Table 1).

\section{Primary outcome measures}

The primary outcome measure was the mean decrease of PASI between the two groups at week 12. The PASI score showed a decrease from baseline until the end of treatment in both groups (see Figure 2A). The mean decrease of PASI at week 12 was $2.03,1.80$ (mean, SD) in the PSORI-CM01 group and $0.89,2.77$ (mean, SD) in the Yinxieling group. The mean decrease of PASI in the PSORI-CM01 group was also greater than that of the Yinxieling group at the end of 3-month follow-up period (see Figure 2A).

\section{Secondary outcome measures}

Secondary outcome measures including BSA, VAS, and
DLQI are also shown in Figure 2. The mean pruritus scores on the VAS at week 12 were $2.49,2.40$ (mean, SD) in the PSORI-CM01 group compared with 3.92, 2.64 (mean, SD) in the Yinxieling group $(\mathrm{t}=-2.261, \mathrm{P}=0.027)$. These results suggested that PSORI-CM01 granules might relieve itching better than Yinxieling tablets (Figure 2C). Moreover, the mean BSA for PV was 7.05, 5.12 (mean, SD) in the PSORICM01 group and 7.70, 7.51 (mean, SD) in the Yinxieling group at week $12(\mathrm{t}=-1.297, \mathrm{P}=0.200)$. The mean DLQI was 5.68, 4.87 (mean, SD) in the PSORI-CM01 group vs. 5.75, 5.02 (mean, SD) in the Yinxieling group at week $12(\mathrm{t}=-0.165, \mathrm{P}=0.869)$. The reduction rate of PASI was calculated as the proportion of reduction of PASI scores after treatment. The results revealed reduction rates of $32 \%$ and $50 \%$ in the PSORI-CM01 group vs. $15 \%$ and $29 \%$ in the Yinxieling group at the end of week 12 and at the 3-month follow-up, respectively.

All secondary outcome measures were decreased from baseline until the end of 3 -month follow-up period in both groups. The data was recorded every 2 weeks in the treatment period and every month in the follow-up period. The repeated measure variance analysis of PASI, reduction of PASI, VAS, DLQI, and BSA showed no significant differences between the two groups at each time point. The curves seemed to exhibit a trend that showed that changes of PASI, VAS, and BSA in the PSORI-CM01 group demonstrated more improvement than those in the Yinxieling group (Figure 2).

\section{Safety}

No abnormal laboratory test results were observed during the entire study period. The proportion of patients that experienced any AEs were similar between the PSORICM01 and Yinxieling groups: 15 of 31 (48.4\%) and 10 of $32(31.3 \%)$, respectively, and the differences were not statistically significant. Only three patients in the PSORICM01 group and one patient in the Yinxieling group discontinued treatment due to AEs and unsatisfactory effect. Most of the AEs reported were mild. Common cold (eight in the PSORI-CM01 group vs. six in the Yinxieling group) was the most commonly reported AE. The percentage of patients experiencing serious or severe adverse events was low and was similar between the two groups. There were two patients in the Affiliated Hospital of Tianjin Academy of Traditional Chinese Medicine who exhibited serious adverse events (SAEs) in the PSORICM01 group. One patient in hospital complained of a sore 
Table 1 Patients' baseline characteristics

\begin{tabular}{|c|c|c|}
\hline Items & PSORI-CM01 group & Yinxieling group \\
\hline Number of subjects & 31 & 32 \\
\hline \multicolumn{3}{|l|}{ Sex, n (\%) } \\
\hline Male & $26(83.9)$ & $21(65.6)$ \\
\hline Female & $5(16.1)$ & $11(34.4)$ \\
\hline Disease duration, years (mean, SD) & $19.33,35.31$ & $10.60,10.78$ \\
\hline PASI, (mean, SD) (min-max) & 13.19, $3.66(10.1-20.4)$ & $11.67,4.17(6.5-17.6)$ \\
\hline BSA for PV (mean, SD) & $7.50,4.00$ & $7.24,5.16$ \\
\hline VAS (mean, SD) & $3.34,2.43$ & $4.00,2.28$ \\
\hline Married & $25(80.1)$ & $28(87.5)$ \\
\hline Unmarried & $6(19.4)$ & $4(12.5)$ \\
\hline \multicolumn{3}{|l|}{ Education, n (\%) } \\
\hline Illiterate & $0(0)$ & $0(0)$ \\
\hline Primary school & $0(0)$ & $4(12.5)$ \\
\hline Middle school & $8(25.8)$ & $6(18.8)$ \\
\hline High school & $9(29.0)$ & $9(28.1)$ \\
\hline College graduate & $14(45.2)$ & $13(40.6)$ \\
\hline \multicolumn{3}{|l|}{ Medical history, n (\%) } \\
\hline With & $31(100.0)$ & $32(100.0)$ \\
\hline Without & $0(0)$ & $0(0)$ \\
\hline
\end{tabular}

CHM, Chinese Herbal Medicine; BMI, body mass index; PASI, Psoriasis Area Severity Index; SD, standard deviation; BSA, body surface area; VAS, pruritus scores on the Visual Analogue Scale; DLQI, Dermatology Life Quality Index.

throat and hoarseness, and the other was hospitalized due to palpitations and chest tightness. Both of them withdrew from the trial and recovered after hospitalization. There were no drug-related SAEs reported. The remaining AEs, such as trauma, contact dermatitis, lower back pain, stomachache, lower limb edema, allergic rhinitis, and retching were limited to a single case in both groups.

\section{Discussion}

This pilot study showed that the full-scale RCT is feasible for randomization, patient recruitment, and assessment. There were 211 patients recruited from the outpatient departments of three centers. The present study also showed that traditional Chinese Medicine monotherapy 

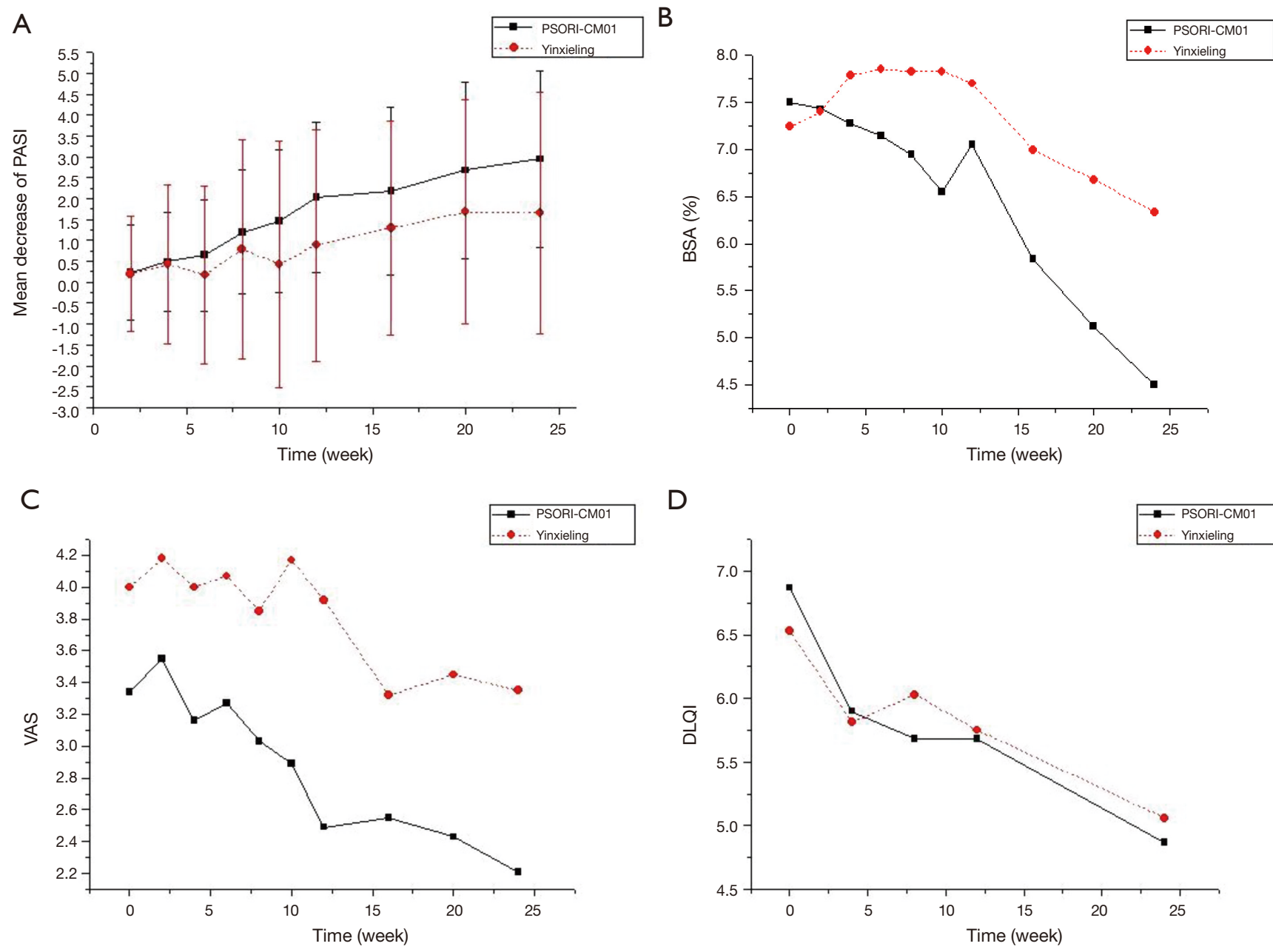

Figure 2 Mean decrease of PASI scores, BSA,VAS and DLQI in the two groups. (A) PASI scores (PASI, Psoriasis Area Severity Index). The PASI score showed a decrease from baseline until the end of treatment in both groups. The mean decrease of PASI at week 12 was 2.03 , 1.80 (mean, SD) in the PSORI-CM01 group and 0.89, 2.77 (mean, SD) in the Yinxieling group. The mean decrease of PASI in the PSORICM01 group was also greater than that of the Yinxieling group at the end of 3-month follow-up period. (B) BSA (body surface area for Psoriasis) scores. The mean BSA also showed a decrease from baseline until the end of treatment in both groups. At week 12, the decrease of BSA was more in PSORI-CM01 group than that of Yinxieling group. (C) VAS (Visual Analogue Scale) scores. The mean pruritus scores on the VAS was less in the PSORI-CM01 group compared with that in the Yinxieling group from baseline to the end of the study. (D) DLQI (Dermatology Life Quality Index) scores. The mean DLQI showed decrease in both groups after treatment but no differences between two groups.

was popular and acceptable for Chinese patients. The Interactive Web Response System for Chinese Medicine Trials (IWRS-CMT) was employed for randomization, and proved to be very convenient to use among different investigators across different centers in a multi-center trial. However, if there will be a patient satisfaction survey, the data would be more convincing. In this pilot study, PSORI-
CM01 granules showed no significant differences compared to Yinxieling tablets in the improvement of PASI, DLQI, and BSA scores after treatment due to the small sample size. However, PSORI-CM01 might have advantages in reducing VAS scores ( $\mathrm{t}=-2.261, \mathrm{P}<0.05$, see Figure $2 C$ ) and seems to exhibit a trend of reducing PASI and acting more effectively than Yinxieling tablets. Changes of PASI scores 
in the PSORI-CM01 group were found to be greater than those in the Yinxieling tablet group from baseline until the end of the 3-month follow-up period. These results were similar to a previous study, which showed that following PSORI-CM01 treatment for 8 weeks, the changes of PASI and DLQI compared with baseline were statistically significant (4). Put simply, PSORI-CM01 granules showed more potential than Yinxieling tablets in the treatment of CPP. So, our hypothesis that PSORI-CM01 granules are superior to Yinxieling Tablet should be verified in a large sample size.

CHM has been used for a long time in China to control psoriasis clinically. However, there are some limitations to its broader usage, which might be related to its drug preparation, complex composition, and unknown mechanism. In ancient China, decoction was considered the primary and key component of the clinical application of CHM; however, the inconvenience of decoction has limited its application in modern times (21). Meanwhile, the complex composition of CHM preparations also makes it difficult to reveal their mechanism in vivo due to the usage of effective constituents that are unclear and sometimes inexplicable. Thus, the development of CHM for the international market is seriously restricted (22).

The PSORI-CM01 formula is developed from only seven herbs; it was optimized from its original formula and was easier than the original to be defined by its chemical profiles. Previous experimental studies have shown that the PSORI-CM01 decoction optimized with the most effective constituents was more effective than the original formula for the inhibition of proliferation of lipopolysaccharide (LPS)stimulated $\mathrm{HaCaT}$ keratinocyte cell line (23). Another experiment using a propranolol hydrochloride-induced psoriasis-like model determined the anti-psoriatic effects of the PSORI-CM01 decoction, and demonstrated that the administration of the PSORI-CM01 formula in low or high doses $(5.98,11.96 \mathrm{~g} / \mathrm{kg}$ ) decreased the Baker scores (24) for pathological morphology assessment. This was similar to the effect of methotrexate, which ameliorated the psoriasislike model in guinea pigs. These results indicate the antipsoriatic effects of the PSORI-CM01 formula in vivo (25).

The PSORI-CM01 granule is a compound made using a PSORI-CM01 decoction that is concentrated and then dried into powder. Compound granules could keep the maximum effective constituents of decoction and they are superior to decoction for clinical applications, such as drug production, storage, and intake (26). Liquid chromatography coupled with an LTQ Orbitrap Mass Spectrometer were used to identify and quantify the characterization of PSORI-CM01 with different dosages. We found that the dominant compounds were the same and the 14 analytes were relatively more stable and higher in the granule preparations than the decoction or tablet preparations (16). For different preparations of PSORICM01, it could be easier to control the drug quality of the granule than the decoction or tablet. Similarly, our previous pilot study also showed no differences in treatment efficacy and safety between the PSORI-CM01 decoction and the granule (Registration number: ChiCTR-IPR-14005281).

This pilot study had some limitations that should be noted. Firstly, it had a higher dropout rate than what we expected, especially in the Hangzhou No. 3 General Hospital. There were 63 patients who participated in the trial, and 24 (9 in the PSORI-CM01 group and 15 in the Yinxieling group) could not finish the study due to moving to other places, dissatisfaction with efficacy, or failure to contact the patients for AEs. Only three of the 24 patients discontinued the trial because of AEs. We recruited patients from summer to autumn and most of the patients withdrew from the study during the Spring Festival. In China, before and after the Spring Festival, which is the most important traditional holiday, Chinese people like to move to other places for business, travel, or go back to their hometowns for an extended period. This might explain the higherthan-expected dropout rate. Therefore, when the full RCT is conducted, we should try to avoid treatment during the Spring Festival when recruiting patients, and consider local patients first to limit the dropout rate. Although we used ITT analysis and the LOCF approach to deal with the missing data, the bias associated with withdrawal and missed clinical data cannot be avoided. Secondly, we had a relatively small sample size, which limited our ability to demonstrate statistical significance in the primary and secondary outcomes. So, a large sample study should be added for verification. Thirdly, the trial had no placebo groups, as it was purely designed to provide evidence regarding the clinical effectiveness of a Chinese medicine treatment for psoriasis before and after optimization and simplification (15). Finally, the treatment duration for 12 weeks might be a bit short for Chinese medicine usually acts on slowly. Further research design is recommended that patients undergo treatment testing for a longer period of time, which is more meaningful and convincing.

In this paper we designed a multicentre, randomized, 
double-blind, double-dummy, pilot study that compared PSORI-CM01 granules with Yinxieling tablets for the treatment of CPP. The study was reported according to the CONSORT (Consolidated Standards of Reporting Trials) 2010 checklist (27). The results showed that it is feasible to expand the pilot trial into a full RCT after implementing some strategies to control the dropout rate. The primary outcome of this study might be less than what we expected in our sample size calculation for conducting a full RCT. At week 12 of this trial, the mean decrease of PASI was 2.03, 1.80 (mean, SD) in the PSORI-CM01 group and 0.89, 2.77 (mean, SD) in the Yinxieling group. In our calculation, we expected an SD of 1.1 in the PSORI-CM01 group and 2.5 in the Yinxieling group. The results of this pilot study should also be taken into account for sample size calculation prior to conducting the RCT. The results indicated that PSORI-CM01 granules and Yinxieling tablets were both safe treatments, and that PSORI-CM01 might offer more advantages than its original type (Yinxieling) and exhibited greater potential to be developed and used widely.

\section{Conclusions}

Our research in this pilot study showed that PSORI-CM01 granules and Yinxieling tablets were both safe and effective treatments that improve psoriasis in patients with CPP. Our results also suggested that PSORI-CM01 granules may decrease PASI and VAS scores more than Yinxieling tablets. Moreover, PSORI-CM01 granules might be more promising for general application. However, considering the high dropout rate in this pilot study, major strategies are necessary before conducting the full-scale RCT. Additional studies with larger sample sizes in a multi-center RCT are also needed.

\section{Acknowledgments}

We thank Prof. Guo-Wei Xuan (Guangdong Provincial Hospital of Chinese Medicine, Guangzhou, China), Prof. Ai-E Xu and Prof. Jun-Jun Shan (The Third Hospital of Hangzhou, Hangzhou, China), and Prof. Jun-ling Zhang and Prof. Hong-mei Wang (Affiliated Hospital of Tianjin Academy of Traditional Chinese Medicine) for their comments, suggestions, and for conducting the study in their centers. We also thank Ms. Mei-Ling Xuan and Ms. Geng Li (Guangdong Provincial Hospital of Chinese Medicine) for the preparation of randomization and allocation.

Funding: This research was supported by the Guangdong Financial Industry Technology Research Development Fund (No. 2011(285)05) and the Traditional Chinese Medicine Bureau Foundation of Guangdong Province (No. 20183005).

\section{Footnote}

Reporting Checklist: The authors have completed the CONSORT reporting checklist. Available at http://dx.doi. org/10.21037/apm-20-2575

Data Sharing Statement: Available at http://dx.doi. org/10.21037/apm-20-2575

Conflicts of Interest: All authors have completed the ICMJE uniform disclosure form (available at http://dx.doi. org/10.21037/apm-20-2575). The authors have no conflicts of interest to declare.

Etbical Statement: The authors are accountable for all aspects of the work in ensuring that questions related to the accuracy or integrity of any part of the work are appropriately investigated and resolved. All procedures performed in this study involving human participants were in accordance with the Declaration of Helsinki (as revised in 2013). The study protocol was approved by the Institutional Ethics Committee of GPHCM (Ethics Statement No.: B2014-026-01, approved on $8^{\text {th }}$ August 2014), the Biomedical Ethics Committee of the Hangzhou No. 3 General Hospital (Ethics Statement No.: B2014-026-01, approved on $8^{\text {th }}$ August 2014), and the Institutional Ethics Committee of the Affiliated Hospital of Tianjin Academy of Traditional Chinese Medicine (Ethics Statement No.: 2014KY-001). All patients provided written informed consent prior to initiation of the study. Trial registration: Chinese Clinical Trial Registry (http://www.chictr.org.cn/index.aspx) ChiCTR-TRC-14005185; date of registration: Aug 8th 2014.

Open Access Statement: This is an Open Access article distributed in accordance with the Creative Commons Attribution-NonCommercial-NoDerivs 4.0 International License (CC BY-NC-ND 4.0), which permits the noncommercial replication and distribution of the article with the strict proviso that no changes or edits are made and the 
original work is properly cited (including links to both the formal publication through the relevant DOI and the license). See: https://creativecommons.org/licenses/by-nc-nd/4.0/.

\section{References}

1. Menter A, Gottlieb A, Feldman SR, et al. Guidelines of care for the management of psoriasis and psoriatic arthritis: Section 1. Overview of psoriasis and guidelines of care for the treatment of psoriasis with biologics J Am Acad Dermatol 2008;58:826-50.

2. Mrowietz U, Kragballe K, Reich K, et al. Definition of treatment goals for moderate to severe psoriasis: a European consensus. Arch Dermatol Res 2011;303:1-10.

3. Zhang LX, Bai YP, Song PH, et al. Effect of Chinese herbal medicine combined with acitretin capsule in treating psoriasis of blood-heat syndrome type. Chin J Integr Med 2009;15:141-4.

4. Yan YH, Lu CJ. Optimized Yinxieling for Treatment of Psoriasis Vulgaris: An Exploratory Clinical Trial. Traditional Chinese Drug Research \& Clinical Pharmacology 2011;22:691-3.

5. May BH, Zhang AL, Zhou W, et al. Oral herbal medicines for psoriasis: a review of clinical studies. Chin J Integr Med 2012;18:172-8.

6. Zhang CS, Yu JJ, Parker S, Zhang AL, et al. Oral Chinese herbal medicine combined with pharmacotherapy for psoriasis vulgaris: a systematic review. Int J Dermatol 2014;53:1305-18.

7. Yu JJ, Zhang CS, Zhang AL, et al. Add-on effect of Chinese herbal medicine bath to phototherapy for psoriasis vulgaris: a systematic review. Evid Based Complement Alternat Med 2013;2013:673078.

8. Dai YJ, Li YY, Zeng HM, et al. Effect of Yinxieling decoction on PASI, TNF- $\alpha$ and IL-8 in patients with psoriasis vulgaris. Asian Pac J Trop Med 2014;7:668-70.

9. Wang L, Huang YJ, Wang MH. Clinical observation of Yinxieling tablets for the treatment of psoriasis vulgaris. J Guanghou Univ TCM 2009;26:520-2.

10. Lu CZ, Wu XX, Liu FN. Effect of Yinxieling on PCNA expression and apoptosis of keratinocyte. Trad Chin Drug Res Clin Pharmacol 2006;17:329-31.

11. Yan YH, Zhao RZ, Lu CJ. Optimization of Yinxieling Capsule with orthogonal design. Lishizhen Medicine and Materia Medica Research 2014;25:2673-5.

12. Jing L, Wang T, Zhou G. Status and thinking about research of further optimization of prescription. Chinese Journal of New Drugs 2013;22:1011-3.
13. Lu C, Deng J, Li L, et al. Application of metabolomics on diagnosis and treatment of patients with psoriasis in traditional Chinese medicine. Biochim Biophys Acta 2014;1844:280-8.

14. Han L, Yu JH, Lou BQ, et al. Effects of YinxielingOptimization-Decoction in treating psoriasis in mouse psoriasis models. IEEE International Conference on Bioinformatics and Biomedicine Workshops 2011:807-10.

15. Deng J, Yao D, Lu C, et al. Oral Chinese herbal medicine for psoriasis vulgaris: protocol for a randomised, doubleblind, double-dummy, multicentre clinical trial. BMJ Open 2017;7:e014475.

16. Chen SD, Lu CJ, Zhao RZ. Identification and quantitative characterization of PSORI-CM01, a Chinese medicine formula for psoriasis therapy, by liquid chromatography coupled with an LTQ Orbitrap mass spectrometer. Molecules 2015; 20:1594-609.

17. Gordon KB, Feldman SR, Koo JY, et al. Definitions of measures of effect duration for psoriasis treatments. Arch Dermatol 2005;141:82-4.

18. Carey W, Glazer S, Gottlieb AB, et al. Relapse, rebound, and psoriasis adverse events: an advisory group report. J Am Acad Dermatol 2006;54:S171-S181.

19. Thabane L, Ma J, Chu R, et al. A tutorial on pilot studies: The what, why and how. BMC Med Res Methodol 2010;10:1.

20. Leon AC, Davis LL, Kraemer HC. The role and interpretation of pilot studies in clinical research. J Psychiatr Res 2011;45:626-9.

21. Zhang HM, Song JZ, Tan HS, et al. From Traditional Decoction to Modern Granule: Perspectives and Prospects of Chinese Medicine Dispensing Granules. World Science and Technology-Modernization of Traditional Chinese Medicine 2012;14:1740-53.

22. Yao XS, Ye WC, Kurihara H. Reveal the scientific connotation of TCM for promoting its modernization and innovative drug research process. Prog Chem 2009;21:2-13.

23. Han L, Peng Y, Zhao R, et al. Effect of Yinxieling Prescription and its modified prescriptions on Keratinocyte $\mathrm{HaCaT}$ proliferation. Journal of Guangzhou University of Traditional Chinese Medicine 2011;28:159-62.

24. Baker BS, Swain AF, Fry L, et al. Epidermal T lymphocytes and HLA-DR expression in psoriasis. Br J Dermatol 1984;110:555-64.

25. Yu JH, Zhao RZ, Lu CJ. Anti-psoriatic effects of optimized Yinxieling Formula on psoriasis-like model of guinea pigs and cell model of lipopolysaccharide-stimulated 
$\mathrm{HaCaT}$ keratinocyte. China Journal of traditional Chinese Medicine and Pharmacy 2013;28:1531-4.

26. Xu YX. Comparison and analysis of the decoction of Chinese medicine and Chinese medicine granules. Journal of Clinical Medical Literature 2015;2:87-8.

27. Moher D, Hopewell S, Schulz KF, et al. CONSORT

Cite this article as: Yao DN, Lu CJ, Wen $\mathrm{ZH}$, Yan YH, Lu LM, Wu HM, He ZY, Deng H, Deng JW. Comparison of PSORI-CM01 granules and Yinxieling tablets for patients with chronic plaque psoriasis: a pilot study for a randomized, doubleblinded, double-dummy, multicentre trial. Ann Palliat Med 2021;10(2):2036-2047. doi: 10.21037/apm-20-2575
2010 explanation and elaboration: updated guidelines for reporting parallel group randomised trials. BMJ 2010; 340:c869.

(English Language Editor: A. Kassem) 